\title{
Histopathological evaluation of carcinoma of breast
}

\author{
Pathak $\mathrm{R}^{1}$, Jha $\mathrm{A}^{2}$, Neupane $\mathrm{PR}^{3}$, Chalise $\mathrm{S}^{2}$, Basnyat $\mathrm{AS}^{2}$ \\ ${ }^{\prime}$ Department of Pathology, Nepal Medical college Teaching Hospital, Jorpati Kathmandu, Nepal. \\ ${ }^{2}$ Department of Pathology, Bhaktapur Cancer Hospital, Bhaktapur, Nepal \\ ${ }^{3}$ Department of Surgery, Bhaktapur Cancer Hospital, Bhaktapur, Nepal.
}

\section{Keywords: \\ Breast carcinoma; Modified Radical \\ Mastectomy; \\ Ductal carcinoma; \\ Medullary features; \\ Lobular; \\ Mucinous.}

\begin{abstract}
Background: Carcinoma of breast has become the major public health problem among females in developing as well as developed countries. In Nepal it comprises $6 \%$ of total cancers cases and often diagnosed at advanced stage. Surgical removal or modified radical mastectomy (MRM) is the most commonly used tools for disease management. The objective of this study is to identify the clinical, macroscopic and microscopic features of MRM specimens.
\end{abstract}

Materials and Methods: This prospective cross-sectional study was carried out at Department of Pathology, Bhaktapur Cancer Hospital, Bhaktapur, Nepal. Macroscopic and microscopic examination provided the tumor size, stage, grade, lymph node status, lympho-vascular invasion and perineural invasion. Data were collected and analyzed using SPSS 16.

Results: The study comprised 112 breast cancer patients of which 109 (97.3\%) were females and 3 $(2.7 \%)$ were males. Invasive ductal carcinoma no specific type was the most common type of breast carcinoma. (84 cases) accounting $75 \%$ of total cases. Carcinoma with medullary features was second most common ( 6 cases) comprising 5.4\% cases followed by lobular, papillary, apocrine, mucinous and NST mixed types. Grade II tumors were most frequent grade observed in $76.79 \%$ cases followed by Grade I (12.50\%) and Grade III (10.71\%).

Conclusion: As a conclusion invasive ductal carcinoma was the most common histological type breast cancer and the tumors were found at T2 and N3 stage i.e maximum at grade II. Our study provides prognostic significance of histo-pathological information in breast cancer management.

\section{INTRODUCTION}

Breast cancer is the most frequently occurring cancer among women in the developed as well as developing

\section{Correspondence:}

Dr. Rakesh Pathak, MD

Department of Pathology,

Nepal Medical College Teaching Hospital, Jorpati, Nepal.

E-mail:dr_rakesh@hotmail.com countries and it has become the major public health problem worldwide with nearly 1.7 million newly diagnosed cases in 2012 representing $25 \%$ of all female cancers. ${ }^{1,2}$ Among Nepalese women, breast cancer is the second most common type of cancer accounting $6 \%$ of total cancers in Nepal. ${ }^{3}$ In Nepal, more than one quarter of the breast cancer is diagnosed in young female and many being diagnosed at an advanced stage with tumors showing more aggressive 


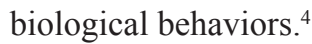

The familial history of breast cancer and mutation of tumor suppressor genes BRCA1, BRCA2 and p53 increases risk of breast cancer. ${ }^{1}$ Alcohol consumption, high dietary fat intake, obesity, overweight, diabetes mellitus and physical inactivity has induced breast cancer incidence. ${ }^{1,2}$ In context to Nepal, breast cancer is commonly diagnosed in female with late menarche, early pregnancy, long lactation period, less breast feeding, change in lifestyle and dietary habits, familial history of breast cancer and contraceptive users. ${ }^{3,5}$

With the rapid increase in breast cancer, tools used for early cancer diagnosis and management in Nepal include breast self examination (BSE), imaging, surgery, chemotherapy, radiation therapy, palliative care and cancer education. ${ }^{3}$ The surgical removal of breast followed by chemotherapy and radiation therapy is the common practices used in disease treatment. A study by Baker et al suggested breast surgery (MRM) for Stage I and II diseases and radical mastectomy for Stage III as the best treatment of choice. ${ }^{6}$

The histopathological factors of breast tumors like tumor size, lymph node status, histological type, histological grade, presence or absence of hormone receptors and age of patients play crucial role on chemotherapy and radiation therapy. ${ }^{7,8}$ The study aims to assess the clinical profile of the patients with breast carcinoma as well as macroscopic and microscopic features of MRM specimens.

\section{MATERIALS AND METHODS}

The present prospective cross-sectional study was carried out at Department of Pathology, Bhaktapur Cancer Hospital, Bhaktapur (BCH), Nepal. A total number of 112 cases that underwent modified radical mastectomy (MRM) in Department of Surgical Oncology, BCH between January 2014 to September 2015 were included in this study. MRM specimens with no residual tumor, patients having previous history of radiotherapy or neo-adjuvant therapy were excluded from the study. The clinical details like age, sex, laterality, mode of tumor presentation, skin and nippleareola changes were assessed. Macroscopic examination provided information regarding size, number and tumor location in mastectomy specimens. Histological type, stage, grade, lympho-vascular invasion, and lymph node involvement were evaluated by microscopic examination of Hematoxylin and Eosin stained tissue sections. Tumor grading and staging were done according to the Nottingham modification of the Scarff Bloom and Richardson's (SBR) grading system and TNM staging [where $\mathrm{T}$ describes the tumor size, $\mathrm{N}$ describes nodular involvement and $\mathrm{M}$ describes distant metastasis]. The tumors were given scores according to Nottingham Prognostic Index (NPI) scoring system. Nottingham modified grading system comprises:
Tubule Formation

Score 1: Majority of tumors ( $>75 \%)$

Score 2: Moderate degree $(10 \%-75 \%)$

Score 3: Little or none $(<10 \%)$

\section{Nulcear Pleomorphism}

Score 1: Small, regular, uniform cells

Score 2: Moderate increase in size and variation

Score 3: Marked nuclear variations

\section{Mitotic Counts}

Score 1: 0-9 Mitoses/10 hpf

Score2: 10-19 Mitoses/10 hpf

Score 3: 20 or $>$ Mitoses/10 hpf

\section{Statistical Analysis}

All the observed data were tabulated in MS-Excel and statistically analyzed using IBM SPSS statistics software, version 16.

\section{RESULTS}

The study comprised a total of 112 breast cancer patients of which $109(97.3 \%)$ were females and $3(2.7 \%)$ were males. The age of the female patients ranged from 27 to 87 years with the mean age of 49.29 years. Majority of female patients were within age group 41 to 60 years (Table 1). The age for male breast cancer patients was between 59 and 84 years with the mean age of 70.67 years. Out of 112 MRM cases, 57 cases involved left breast whereas, 55 involved right breast and no any bilateral breast involvement was observed.

In our study, invasive ductal carcinoma was the most commonly observed histological type of breast cancer with 84 cases (Table 2). Carcinoma with medullary carcinoma was the second most observed type followed by lobular, papillary, apocrine, mucinous and NST mixed types. In

Table 1: Age and sex distribution of breast cancer patients

\begin{tabular}{ccc}
\hline Age (Years) & Female & Male \\
\hline $21-40$ & 26 & 0 \\
$41-60$ & 65 & 1 \\
$61-80$ & 17 & 1 \\
$>81$ & 1 & 1 \\
Total & $\mathbf{1 0 9}$ & $\mathbf{3}$
\end{tabular}


Table 2: Incidence of various histologic types of breast neoplasm

\begin{tabular}{|c|c|c|c|c|}
\hline \multirow{2}{*}{ S/No. } & \multirow{2}{*}{ Type of neoplasm } & \multicolumn{2}{|c|}{ Sex } & \multirow{2}{*}{ Number of Cases (\%) } \\
\hline & & Male & Female & \\
\hline 1. & Invasive Ductal Carcinoma (NST: fig.3) & 2 & 82 & $84(75 \%)$ \\
\hline 2. & Carcinoma with medullary features (fig.1) & - & 6 & $6(5.4 \%)$ \\
\hline 3. & Lobular Carcinoma (fig.2) & - & 4 & $4(3.6 \%)$ \\
\hline 4. & Invasive Papillary Carcinoma & 1 & 2 & $3(2.6 \%)$ \\
\hline 5. & NST with Apocrine Differentiation & - & 3 & $3(2.6 \%)$ \\
\hline 6. & Apocrine Carcinoma & - & 2 & $2(1.8 \%)$ \\
\hline 7. & Micropapillary Carcinoma (fig.4) & - & 2 & $2(1.8 \%)$ \\
\hline 8. & Mucinous Carcinoma (fig.5) & - & 2 & $2(1.8 \%)$ \\
\hline 9. & NST with Mucinious Component & - & 2 & $2(1.8 \%)$ \\
\hline 10. & Mixed NST and Lobular Carcinoma & - & 2 & $2(1.8 \%)$ \\
\hline \multirow[t]{2}{*}{11.} & Mixed NST and Micropapillary Carcinoma & - & 2 & $2(1.8 \%)$ \\
\hline & Total & 3 & 109 & $112 /(100 \%)$ \\
\hline
\end{tabular}

Table 3: Histological grading of breast tumor based on modified SBR system

\begin{tabular}{ccc}
\hline Degree of differentiation & $\begin{array}{c}\text { Nottingham } \\
\text { Grade (Score) }\end{array}$ & $\begin{array}{c}\text { Number of } \\
\text { Cases }\end{array}$ \\
Well differentiated & I $(3-5)$ & 14 \\
Moderately differentiated & II $(6-7)$ & 86 \\
Poorly differentiated & III $(8-9)$ & 12 \\
\hline
\end{tabular}

male, 2 cases were of invasive ductal carcinoma NST and 1 case was of invasive papillary carcinoma.

In the present study we found 14 cases were of Grade I, 86 cases were of Grade II and 12 cases were of Grade III as shown in Table 3. Grade II tumors were most frequent comprising $76.79 \%$ cases followed by Grade I (12.50\%) and Grade III (10.71\%). In the present study we found maximum $75(67 \%)$ cases were of T2 stage $(2-5 \mathrm{~cm})$ and the frequency of the tumors were T3,T1,T4 respectively. Graph 1 represents the TNM staging based on axillary lymph nodes involvement. In this study we observed majority of cases were at $\mathrm{N} 3$ stage involving more than 10 lymph nodes followed by N0, N2 and N1.

\section{DISCUSSION}

Breast cancer is the top cancer in female population worldwide and incidence has increased at the rate of $3-4 \%$ in developing countries and often gets diagnosed at late stage. ${ }^{9}$ According to a study conducted in Central Nepal, breast cancer had high prevalence among reproductive cancers and the susceptible age group was 40 to 50 years female. ${ }^{10}$ In this study we found female aged between 41 to 60 years had higher prevalence of breast cancer as compared to other age group. A similar study by Acharya et al observed the most common age group to be diagnosed with breast cancer

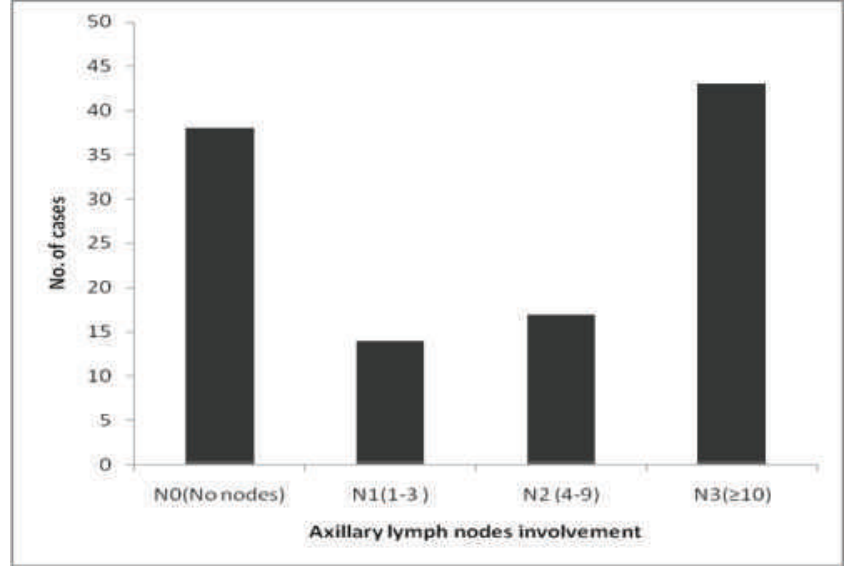

Graph 1: TNM staging based on the Axillary lymph node status

was 41 to 55 years. ${ }^{7}$ In a study the mean age of disease get diagnosed was 49.35 years. ${ }^{9}$ The incidence of male breast cancer is very rare but we found 3 cases accounting $2.6 \%$ of total cases. The result was in accordance to a study conducted by Rai et al who found $0.5 \%$ incidence of male breast cancer. ${ }^{11}$

In the present study invasive ductal carcinoma NST was the mostly observed histological type of breast carcinoma accounting $75 \%$ of the total cases. A clinical database study conducted by Wang et al reported highest $90.1 \%$ of breast carcinoma was invasive ductal type similar to our study. ${ }^{12}$ A study by Reddy et al observed $85.05 \%$ cases with duct cell carcinoma NOS type which correlates with our study. ${ }^{13}$ Similar studies reported maximum frequency of breast cancer cases were of invasive ductal carcinoma type. ${ }^{14-}$ ${ }^{17}$ Kakarala et al reported Asian Indian/Pakistani women had more invasive ductal carcinoma and less invasive lobular carcinoma as compared to Caucasians. ${ }^{18}$ In male patients, we observed invasive ductal carcinoma was the 


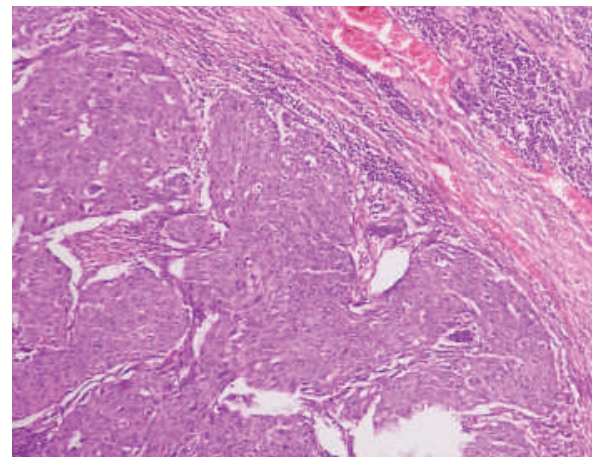

Figure 1: Carcinoma with medullary features (HE stain, X200)

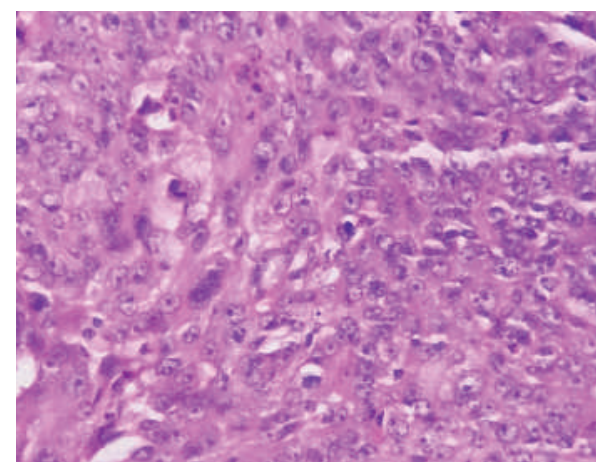

Figure 3: Infiltrating breast carcinoma of no special type, poorly differentiated showing absence of tubules, marked pleomorphism and prominent mitotic figures (HE stain, X400)

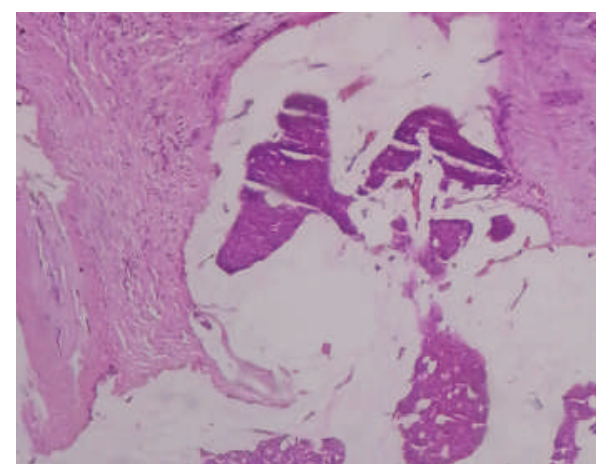

Figure 5: Mucinous carcinoma of breast (HE stain, X200)

most common type of breast cancer followed by invasive papillary. Among 84 cases invasive ductal carcinoma, two were male patients. A study performed at PGIMER by Rai et al reported infiltrating ductal carcinoma was most common type of breast cancer in male and invasive papillary was second most common and the results were similar to our study. ${ }^{11}$

In this study we found medullary carcinoma was the second common histological type, observed in 6 cases $(5.4 \%)$ followed by lobular carcinoma in 4 cases (3.6\%). In contrast to our study, lobular carcinoma was found to be second

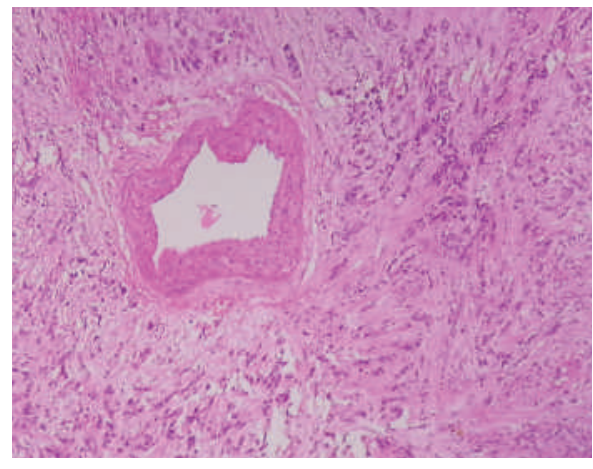

Figure 2: Lobular carcinoma of breast showing single file pattern (HE stain, X200)

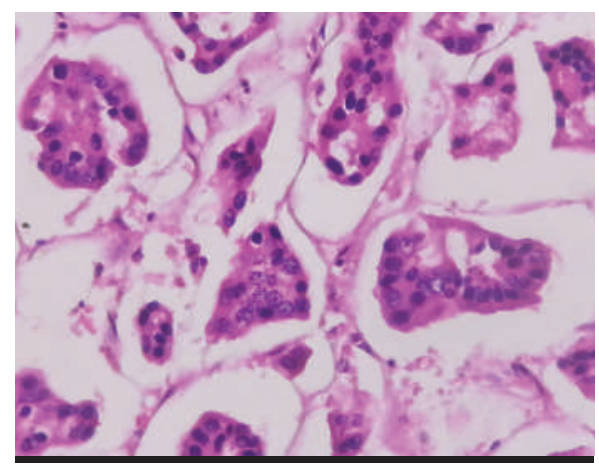

Figure 4: Micropapillary carcinoma of breast (HE stain, X400)

most frequent type of breast cancer. ${ }^{12,15}$ We observed 3 cases of invasive papillary carcinoma, out of which one was male case. Similarly we identified 3 cases of NST with apocrine differentiation and 2 each cases of apocrine, micropapillary, mucinous, NST with mucinous, NST mixed with lobular and micropapillary carcinoma type.

Histological grading are based on Nottingham modification of the Scarff Bloom and Richardson's grading system. The extent of tubule formation, nuclear size and pleomorphism, and mitotic rate are the parameters measured. Each of the three elements was assigned with a score 1 to 3 and the final grade was identified from the sum of each individual scores. Depending on the degree of differentiation, well differentiated (grade I) scores 3 to 5, moderately differentiated (grade II) scores 6 to 7 and poorly differentiated (grade III) scores 8 to $9 .{ }^{19}$

In the present study we found majority of tumors were moderately differentiated grade II accounting 86 (76.79\%) of total cases followed by $14(12.50 \%)$ tumors with well differentiated grade I and $12(10.71 \%)$ tumors with poorly differentiated grade III. Reddy et al found significantly higher percentage of grade II tumors that was similar to our study. ${ }^{13}$ Similarly, Acharya et al observed grade II tumors were most frequent comprising $47.40 \%$ of total tumors following grade I and grade III that are in accordance with our findings. ${ }^{7}$ 
According to the AJCC TNM staging criteria, the tumor size varied from $1.0 \mathrm{~cm}$ to $9.0 \mathrm{~cm}$, with majority $(67.0 \%)$ of tumors belonging to T2 $(2.0$ to $5.0 \mathrm{~cm})$ stage, followed by T3 (more than $5 \mathrm{~cm}$ ), T1 (Less than $2 \mathrm{~cm}$ ) and T4 (tumor growing to chest wall or skin). A study observed $47.72 \%$ cases with T2 stage that correlate with our results. ${ }^{14}$ Similarly, other studies also observed highest frequency of tumors with $\mathrm{T} 2$ stage. ${ }^{7,18}$

In this study highest frequency of tumors belonged to N3 stage (38.4\%) involving more than 10 nodes followed by N0 stage (no nodes involved), N2 stage (4 to 9 nodes involved) and $\mathrm{N} 1$ stage (1 to 3 nodes involve. In contrast to our study, other studies have shown $\mathrm{N} 0$ as the most common stage followed by N1,N2,N3 respectively. ${ }^{9,12,13,18}$

In addition, we found $70(62.5 \%)$ cases had lymphovascular invasion and 49(43.8\%) cases had perineural invasion. A study by Song et al reported 54.2\% MRM cases i.e. maximum percentage of breast carcinoma had lymphovasular invasion similar to our result. ${ }^{20}$

\section{CONCLUSION}

The present study has provided the information about the histo-pathological aspects of breast cancer patients diagnosed at $\mathrm{BCH}$, Bhaktapur. Invasive ductal carcinoma (NST) was the most common histological type breast cancer. Majority of patients were diagnosed with tumor at T2 and N3 stage i.e maximum at grade II. Our study puts prognostic significance of histo-pathological information in treatment planning and strategies.

\section{Acknowledgement}

We would like to acknowledge all the staffs of Department of Pathology and Surgery, BCH who helped us in this research work.

\section{REFERENCES}

1. World Health Organization. Breast cancer: prevention and control. Switzerland: World Health Organization; 2015 Mar [cited 2016 Jan 10] Available: Crossref

2. World Cancer Research Fund International. Breast cancer statistics. Lyon, France: International Agency for Research on Cancer; 2014 [cited 2016 jan 10] Available: Crossref

3. Singh YP, Sayami P. Management of breast cancer in Nepal. J Nepal Medical Assoc 2009;48:252-7. Crossref

4. Thapa B, Singh YP, Sayami P, Shrestha UK, Sapkota R, Sayami G. Breast cancer in young women from a low risk population in Nepal. Asian Pac J Cancer Prev 2013;14:50959. Crossref

5. Yadav NK, Poudel B, Thanpari C, Koner BC. Assessment of biochemical profiles in premenopausal and postmenopausal women with breast cancer. Asian Pac J Cancer Prev 2012;13:3385-8. Crossref

6. Baker RR, Montague ACW, Childs JN. A comparison of modified radical mastectomy to radical mastectomy in the treatment of operable breast cancer. Ann.Surg 1978; 553-7.

7. Acharya SC, Jha AK, Manandhar T. Clinical profile of patients with breast cancer in Nepal. Kathmandu Univ Med J 2012;39:3-7.

8. Shah K, Raychaudhuri G, Chattopadhyay BK. Clinicopathological study of breast carcinoma: A prospective twoyear study in a tertiary care hospital. Clin Cancer Investig J 2013;2:34-40. Crossref

9. Afsharfard A, Mozaffar M, Orang E, Tahmasbpour E. Trends in epidemiology, clinical and histopathological characteristics of breast cancer in Iran: results of a 17 year study. Asian Pac J Cancer Prev 2013;14:6905-11. Crossref

10. Khan GM, Thapa RK, Adhikari DS, Rajbhandari M, Dwa P, Shrestha S, Oli S. Cancer prevalence trend in central region of Nepal. Journal of Chitwan Medical College 2013;3:22-5. Crossref

11. Rai B, Dip NB, Ghosal S, Sharma SC. Breast cancer in males: a PGIMER experience. J Cancer Res Ther 2005;1:31-3. Crossref

12. Wang LW, Yang GF, Chen JM, Yang F, Yuan JP, Sun SR, Chen $\mathrm{C}, \mathrm{Hu} \mathrm{MB}$, Li Yan. A clinical database of breast cancer patients reveals distinctive clinico-pathological characteristics: a study from central china. Asian Pac J Cancer Prev 2014;15:1621-6. Crossref

13. Reddy SR, Chaitanya B, Latha PS. Histopathological prognostic factors in carcinoma breast- An Indian institutional experience.Int J Med Res Rev 2014;2:573-7.

14. Siddiqui MS, Kayani N, Sulaiman S, Hussainy AS, Shah SH, Muzaffar S. Breast carcinoma in Pakistani females: A morphology study of 572 breast specimens. JPMA 2000;50:18.

15. Albreksten G, Heuch I, Thoresen SO. Histological type and grade of breast cancer tumors by parity, age of birth, and time since birth: a register-based study in Norway. BMC Cancer 2010;10:1-11.

16. Dauda AM, Misauno MA, Ojo EO. Histopathological types of breast cancer in Gombe, North Eastern Nigeria: A sevenyear review. Afr J Reprod Health 2011;15:107-9.

17. Ozmen V. Breast cancer in Turkey: clinical and histopathological characteristics (Analysis of 13,240 patients). J Breast Health 2014;10:98-105. Crossref 
18. Kakarala M, Rozek L, Cote M, Liyanage S, Brenner DE. Breast cancer histology and receptor status characterization in Asian Indian and Pakistani women in the U.S.-a SEER analysis. BMC Cancer 2010;10:1-8. Crossref

19. Elston CW, Ellis IO. Pathological prognostic factors in breast cancer I. The value of histological grade in breast cancer : experience from a large study with long-term follow-up. Histopathology 1991;19:403-10. Crossref
20. Song YJ, Shin SH, Cho JS, Park MH, Yoon JH, Jegal YJ. The role of lymphovascular invasion as a prognostic factor in patients with lymph nodes-positive operable invasive breast cancer. J Breast Cancer 2011;14:198-203. Crossref 